\title{
Image-derived and physiological markers to predict adequate adenosine-induced hyperemic response in Rubidium-82 myocardial perfusion imaging
}

Martin Lyngby Lassen, PhD, ${ }^{\mathrm{a}}$ Mads Wissenberg, MD, PhD, ${ }^{\mathrm{b}}$ Christina Byrne, MD, PhD, ${ }^{a}$ Majid Sheykhzade, PhD, ${ }^{c}$ Preetee Kapisha Hurry, MD, ${ }^{a}$ Anne Vibeke Schmedes, ${ }^{d}$ Andreas Kjær, MD, PhD, DMSc, ${ }^{a}$ and Philip Hasbak, $M D, \mathrm{DMSc}^{\mathrm{a}}$

a Department of Clinical Physiology, Nuclear Medicine \& PET and Cluster for Molecular Imaging, of Biomedical Sciences, Section 4011, Rigshospitalet, University of Copenhagen, Copenhagen, Denmark

b Department of Cardiology, Copenhagen University Hospital, Gentofte, Denmark

c Department of Drug Design and Pharmacology, Faculty of Health and Medical Sciences, University of Copenhagen, Copenhagen, Denmark

d Department of Biochemistry and Immunology, Lillebaelt Hospital, Vejle, Denmark

Received Oct 13, 2021; accepted Dec 22, 2021

doi: $10.1007 / \mathrm{s} 12350-022-02906-9$

Aims. This study aimed to investigate the potential of different markers to identify adequate stressing in subjects with and without caffeine intake prior to Rubidium-82 myocardial imaging.

Methods and Results. This study comprised 40 healthy subjects who underwent four serial Rubidium-82 rest/adenosine stress MPI; two with 0mg caffeine consumption (baseline MPIs) and two with controlled consumption of caffeine (arm 1: 100 and 300mg, or arm 2: 200 and $400 \mathrm{mg}$ ). We report the sensitivity and specificity of seven markers ability to predict adequate adenosine-induced hyperemic response: (1) the splenic response ratio (SRR); (2) splenic stressto-rest intensity ratios (SIR); (3) changes in heart rate $(\Delta \mathrm{HR}) ;(4)$ percentwise change in heart rate $(\triangle \% \mathrm{HR}) ;(5)$ changes in the rate pressure product $(\triangle \mathrm{RPP}) ;(6)$ changes in the systolic blood pressure $(\triangle \mathrm{SBP})$; and $(7)$ changes in the cardiovascular resistance $(\triangle \mathrm{CVR})$. Adequate stressing was determined as stress myocardial blood flow $>3 \mathrm{ml} / \mathrm{g} / \mathrm{min}$ and a corresponding myocardial flow reserve $>68 \%$ of the individual maximum myocardial flow reserve obtained in the baseline MPIs.

Results. 129 MPI sessions (obtained in 39 subjects) were considered for this study. The following sensitivities were obtained: $\mathrm{SSR}=72.7 \%, \mathrm{SIR}=63.6 \%, \Delta \mathrm{HR}=45.5 \%, \Delta \% \mathrm{HR}=$ 77.3\%, $\Delta \mathrm{RPP}=\mathbf{5 4 . 5 \%}, \Delta \mathrm{SBP}=\mathbf{4 7 . 7 \%}$, and $\Delta \mathrm{CVR}=\mathbf{4 0 . 9 \%}$, while the specificities were SSR $=$ $80.9 \%, \mathrm{SIR}=85.0 \%, \Delta \mathrm{HR}=90.4 \%, \Delta \% \mathrm{HR}=81.6 \%, \Delta \mathrm{RPP}=81.1 \%, \Delta \mathrm{SBP}=86.4 \%$, and $\triangle \mathrm{CVR}=90.4 \%$.

Supplementary Information The online version contains supplementary material available at https://doi.org/10.1007/s12350-02202906-9.

The authors of thisarticle have provided a PowerPoint file, available for download atSpringerLink, which summarises the contents of the paper and is freefor re-use at meetings and presentations. Search for the article DOIon SpringerLink.com.
Reprint requests: Martin Lyngby Lassen, PhD, Department of Clinical Physiology, Nuclear Medicine \& PET and Cluster for Molecular Imaging, of Biomedical Sciences, Section 4011, Rigshospitalet, University of Copenhagen, Blegdamsvej 9, 2100 Copenhagen, Denmark; martin.lyngby.lassen@regionh.dk

J Nucl Cardiol 2022;29:3207-17.

1071-3581/\$34.00

Copyright (c) 2022 The Author(s) 
Conclusion. The image-derived and physiological markers all provide acceptable sensitivities and specificities when patients follow the caffeine pausation before MPI. However, their use warrants great care when caffeine consumption cannot be ruled out. ( $\mathrm{J}$ Nucl Cardiol 2022;29:3207-17.)

Key Words: Myocardial flow reserve • Cardiac PET • 82-Rubidium • Pharmacological stress - Adenosine

\begin{tabular}{|ll|}
\hline $\begin{array}{l}\text { Abbreviations } \\
\Delta \mathrm{CVR}\end{array}$ & $\begin{array}{l}\text { Changes in the cardiovascular resis- } \\
\text { tance between rest and stress MPI }\end{array}$ \\
$\Delta \mathrm{HR}$ & $\begin{array}{l}\text { Changes in heart rate between rest and } \\
\text { stress MPI }\end{array}$ \\
$\mathrm{MFR}$ & Myocardial flow reserve \\
$\mathrm{MPI}$ & Myocardial perfusion imaging \\
$\mathrm{PCC}$ & Plasma caffeine concentration \\
${ }^{82} \mathrm{Rb}$ & 82-Rubidium \\
$\Delta \mathrm{RPP}$ & Changes in the rate pressure product \\
& between rest and stress MPI \\
$\mathrm{SIR}$ & Splenic stress-to-rest intensity ratio \\
$\mathrm{SRR}$ & Splenic response ratio \\
\hline
\end{tabular}

See related editorial, pp. 3218-3220

\section{INTRODUCTION}

Myocardial perfusion imaging (MPI) is a common test, where Rubidium-82 $\left({ }^{82} \mathrm{Rb}\right)$ has become the most used tracer in Positron Emission Tomography studies as it can be used in centers without cyclotrons. ${ }^{1,2}$ The accuracy of the stress myocardial blood flow and reserve assessments, however, is significantly lowered if adequate pharmacological stressing is not achieved. ${ }^{3}$ Previous studies have suggested that $3-20 \%$ of all stress MPI might have false-negative findings caused by inadequate pharmacological stressing of the patients. ${ }^{3-5}$ The most frequently used pharmacological stressors are regadenoson, dipyridamole, and adenosine; they all activate the adenosine receptor $\mathrm{A}_{2 \mathrm{~A}}$ in coronary arteries and initiate a dilatation of these vessels, which in turn increases myocardial blood flow up to fourfold. ${ }^{6}$ Coffee, caffeine, and other methylxanthines act as non-selective antagonists at the adenosine receptors and are expected to dose-dependently attenuate vasodilator-induced myocardial hyperaemia and may, therefore, reduce the sensitivity of radionuclide MPI for the detection of inducible perfusion abnormality in patients with coronary artery disease. ${ }^{7}$ The easy identification of adequate hemodynamic response in the patients persists to be a problem; however, several physiological, imagederived, and hybrid markers have been suggested to identify the adequate response. ${ }^{3,8,9}$ This study aimed to evaluate seven of the proposed markers ability to predict adequate adenosine-induced hyperemic response in subjects with or without caffeine consumption prior to MPI. The seven markers can be classified as image- derived markers (splenic response ratio (SRR) and splenic stress-to-rest intensity ratios (SIR)) and markers of changes in physiological response between rest and stress MPI (heart rate $(\Delta \mathrm{HR})$, the percentwise change in $\mathrm{HR}(\Delta \% \mathrm{HR})$, changes in the rate pressure product $(\triangle \mathrm{RPP})$, and changes in the systolic blood pressure $(\triangle \mathrm{SBP}))$. Finally, we evaluate a hybrid marker employing both image data and physiological measures (changes in the cardiovascular resistance between rest and stress MPI ( $\triangle \mathrm{CVR})$ ).

\section{MATERIALS AND METHODS}

\section{Study Population}

This study comprised 40 young, healthy subjects (median age $=23$, interquartile range $=[22,25], 19$ females) recruited for ${ }^{82} \mathrm{Rb}$ rest/stress MPI in a hybrid PET/CT system. Each subject had four serial MPI sessions within 27 days (interquartile range $=17-36$ days), acquired with and without controlled caffeine consumption before the MPI session. Inclusion criteria were age $>18$ years, no participation in studies testing drugs, no regular consumption of medicine, no known medical condition, and no use of tobacco and euphoric substances (except alcohol) within three months prior to study participation. Exclusion criteria were pregnancy, allergy, intolerance to theophylline or adenosine, any prior medical history of asthma, and inability to adhere to the study protocol. The Scientific Ethics Committee of the Capital Region of Denmark [protocol number H15009293] and the Danish Data Protection Agency approved this study, and all volunteers provided informed oral and written consent.

\section{Imaging Protocol}

Positron emission tomography acquisition The subjects were divided into two groups, both groups having four serial ${ }^{82} \mathrm{Rb}$ MPI sessions, each consisting of an ${ }^{82} \mathrm{Rb}$ rest/stress imaging protocol (Figure 1). For each acquisition, the subjects had a target injection dose of $1,100 \mathrm{MBq}(30 \mathrm{mCi}){ }^{82} \mathrm{Rb}$ in a 128 slice positron emission tomography/computed tomography system (Siemens Biograph mCT). Pharmacological stressing was obtained using adenosine infused at $140 \mathrm{mg} / \mathrm{kg} / \mathrm{min}$ for six minutes, with the emission acquisition starting 2.5 minutes into the infusion. The 


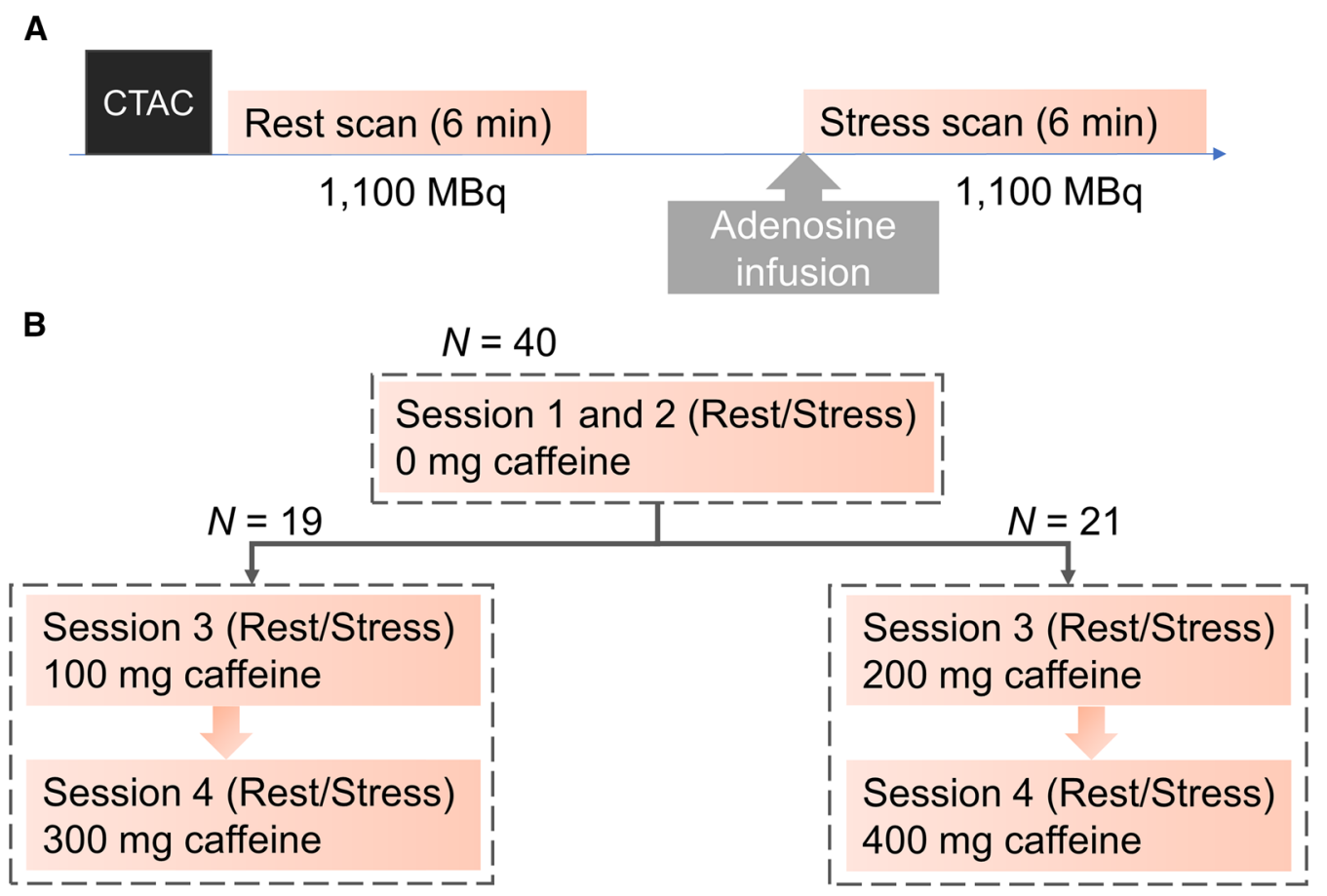

Figure 1. a MPI protocol for each of the 4 PET/CT MPI sessions. b Study protocol for the 40 healthy subjects. PET positron emission tomography, $C T$ computed tomography, CTAC CT attenuation correction.

subjects were instructed to abstain from caffeine at least 24 hours before each of the four MPI sessions to comply with the controlled caffeine consumption setup. Each study subject had two MPI sessions obtained without controlled caffeine intake before the MPI sessions (baseline MPIs) and two with controlled caffeine intake. For the two MPI sessions with controlled caffeine intake, one half had an oral intake of $100 \mathrm{mg}$ and 300 caffeine (arm 1), while the other half had an oral intake of $200 \mathrm{mg}$ and $400 \mathrm{mg}$ caffeine (Figure 1). For the controlled caffeine intake MPI's, the caffeine was provided as tablets diluted into hot water, taken orally $1 \mathrm{hr}$ before the rest acquisition. Plasma caffeine concentrations (PCC) were evaluated at the time of the stress MPI by averaging measurements obtained at 75 and 90 min post ingestion of caffeine, using high-performance liquid chromatography-mass spectrometry (LC-MS/ MS).

Positron emission tomography reconstruction protocol and data processing Four image series were employed in the study; two dynamic image series used for assessments of myocardial blood flow and reserve, and two static image series used for ratio analyses (SRR, SIR, and $\triangle \mathrm{CVR}$ ). The dynamic image series were reconstructed into 18 frames $(1 \times 10 \mathrm{~s}$,
$8 \times 5 s, 3 \times 10 s, 2 \times 20 s, 4 \times 60 s)$ using an iterative 3D ordered subset expectation maximization model with corrections for time-of-flight and point-spread function employing 2iterations, 21subsets. The static images were reconstructed utilizing data from 3.5 minutes, using an injection-to-scan delay of $2.5 \mathrm{~min}$. Both image series had a $6.5 \mathrm{~mm}$ Gaussian post filtering of the data. Myocardial blood flow was calculated using the Lortie model,${ }^{10}$ while the myocardial flow reserve (MFR) was calculated as the ratio between the rest and stress blood flows. Both myocardial blood flow and MFR values were obtained in dedicated software (QPET, CedarsSinai Medical Center, Los Angeles, California, USA).

Image-derived markers SRR was calculated using a previously described method, employing static rest and stress ${ }^{82} \mathrm{Rb}$ perfusion images ${ }^{11}$ (Eq. 1).

$$
S S R=\frac{\text { Stress }_{\text {Spleen }} / \text { Stress }_{\text {Liver }}}{\text { Rest }_{\text {Spleen }} / \text { Rest }_{\text {Liver }}}
$$

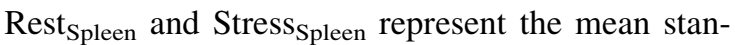
dardized uptake values obtained in the spleen at rest and stress using a $20 \mathrm{~mm}$ spherical volume of interest (VOI), respectively; Rest $t_{\text {Liver }}$ and Stress Liver $_{\text {represent the mean }}$ rest and stress standardized uptake values obtained in the liver using a $50 \mathrm{~mm}$ spherical VOI (Figure 2). ${ }^{12}$ The 
normal limits of SRR were calculated using a previously described method, using the mean +1 standard deviation (SD). ${ }^{12}$ Only baseline MPIs with PCC $<1.0 \mathrm{mg} / 1$ were considered to ensure the normal range was representative for the guideline-recommended withdrawal of caffeine before stress MPI sessions. In this study, $\mathrm{SRR} \leq 0.75$ were considered to have splenic activation (group average $\mathrm{SRR}=0.59, \mathrm{SD}=0.16$ ).

Using the same segmentations, SIR was calculated by normalizing the stress ${ }^{82} \mathrm{Rb}$ uptake observed in the spleen to the activity observed during rest (Eq. 2):

$$
S I R=\frac{\text { Stress }_{\text {Spleen }}}{\text { Rest }_{\text {Spleen }}}
$$

The cut-off value was obtained using an average SIR obtained for the same baseline MPIs + 1SD as used for the SRR calculations. SIR $\leq 0.83$ were considered to have adequate stressing (group average $\mathrm{SIR}=0.70, \mathrm{SD}$ $=0.13)$.
Physiological markers The cut-off values obtained for the physiological markers all rely on measurements obtained for the baseline scans, with $\mathrm{PCC}<1.0 \mathrm{mg} / \mathrm{L}$.

Peak HR for rest and stress scans using the ECG trigger signals were obtained by averaging the three fastest consecutive beats obtained using the three lead ECG-triggering system. Increases in the peak heart rate $(\Delta \mathrm{HR}) \geq 18 \mathrm{~min}^{-1}$ were considered a marker for adequate stressing of the subjects, calculated as the average increase in $\mathrm{HR}-1 \mathrm{SD}$ (average increase in $\mathrm{HR}=$ $29, \mathrm{SD}=11)$. In addition, a percentwise increase in the $\mathrm{HR}$ from rest to stress was reported $(\Delta \% \mathrm{HR})$ using a cutoff value of $25 \%$, calculated as the average percentwise increase $-1 \mathrm{SD}$ (average change $=43 \%$, SD $=18 \%$ ).

Rate pressure products (RPP) were calculated for all MPI sessions defined as RPP = systolic blood pressure)*(Heart rate). Increases in the RPP $\geq 1,250 \mathrm{mmHg}$ * $\min ^{-1}$ from rest to stress MPI was considered a marker
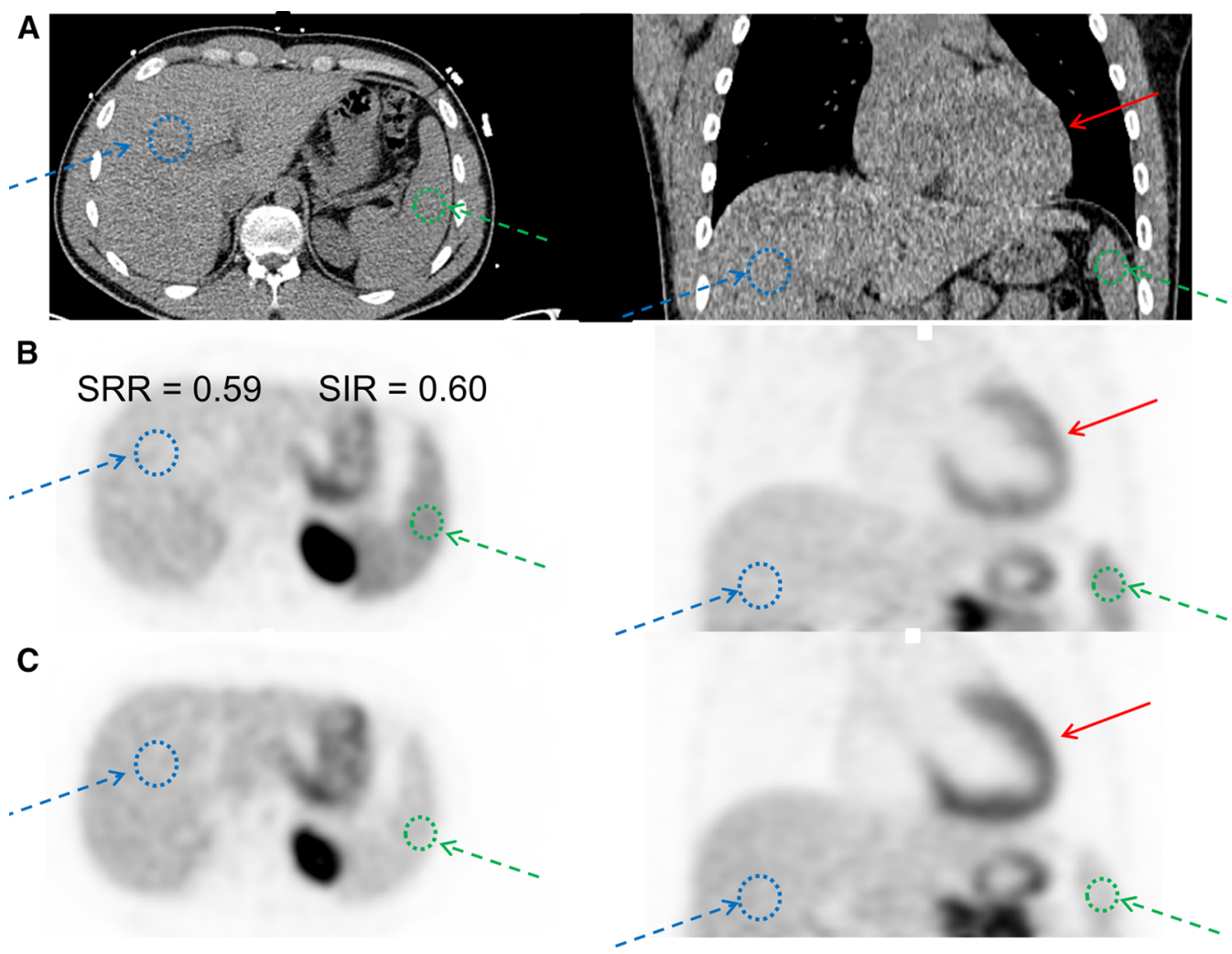

Figure 2. Identification of SRR and SIR. Two volumes of interest were in the spleen (sphere, $r=$ $20 \mathrm{~mm}$ - green arrow) and liver (sphere, $r=50 \mathrm{~mm}$ - blue arrow). Anatomical identification of the spleen and heart (red arrow) was obtained using the low-dose CT images acquired for attenuation correction purposes (a). In $\mathbf{b}$ and $\mathbf{c}$ we show the rest and stress uptake parameters for a scan with concordant assessment of adequate hyperemic response as obtained using both SRR and SIR. SRR splenic response ratio, SIR splenic stress-to-rest intensity ratio. 
for adequate stress using a cut-off value determined as the mean change in RPP - $1 \mathrm{SD} ; \Delta \mathrm{RPP} \geq 1250$ (average $=3012, \mathrm{SD}=1733$ ).

Changes in the systolic blood pressure $(\triangle \mathrm{SBP})$ were calculated for all MPI sessions defined as the systolic rest blood pressure - systolic stress blood pressure. Decreases in the systolic blood pressure $\geq 38 \mathrm{mmHg}$ from rest to stress MPI was considered a marker for adequate stress using a cut-off value determined as the mean change in the systolic pressure - $1 \mathrm{SD} ; \Delta \mathrm{SBP} \geq 38$ (average $=48, \mathrm{SD}=10$ ).

Hybrid markers Cardiovascular resistance was obtained for all MPIs using Eq. 3.

$C V R=0.33$

$\times \frac{(2 \times \text { diastolic blood pressure }+ \text { systolic blood pressure })}{\text { Myocardialbloodflow }}$

For the rest MPI sessions, diastolic and systolic blood pressures were acquired immediately before the emission acquisition, while they were measured 2-2.5 min into the adenosine infusion and, thus, immediately before ${ }^{82} \mathrm{Rb}$ infusion start for the stress MPI sessions. The cut-off for normal changes in the cardiovascular resistance $(\triangle \mathrm{CVR})$ was considered as mean $\triangle \mathrm{CVR}-$ 1SD obtained for baseline MPIs with repeat PCC $<1.0 \mathrm{mg}$, introducing a cut-off on $40 \%$ reduction (mean $=68 \%, \mathrm{SD}=28)$. The unit for the cardiovascular resistance is $\mathrm{mmHg} / \mathrm{mL} / \mathrm{g} / \mathrm{min}$.

MFR: normal range and reproducibility The healthy subjects were expected to have normal MFR ( $\geq$ $2.5)$; thus, the usual thresholds for normal flow reserve could not be used. ${ }^{13}$ To ameliorate the anticipated increased MFR values, we introduced a populationbased cut-off level based on the hyperemic responses obtained in patients with both repeat scans PCC $<1.0$ mg. For these patients, we normalized the repeated MFR values to the intra-subject highest MFR. Using only interscan variation values, we obtained the normal interscan variation in MFR defined as the average interscan difference for the baseline MPIs - 1SD (average off-set $=83.0 \%, \mathrm{SD}=15.0 \%$; cut-off $=$ $68 \%$ ). In this context, sufficient hyperemic response was only determined with stress myocardial blood flow $>$ $3 \mathrm{ml} / \mathrm{g} / \mathrm{min}$ and MFR $\geq 68 \%$ of the individual maximum obtained MFR during the baseline scans.

Sensitivity, specificity, and positive and negative predictive values were calculated for all seven markers using confusion matrices. In brief, the sensitivity is calculated as (true positive measures)/((true positive measures) + (false-negative measures)), specificity is calculated as (true negative measures)/(true negative measures $)+($ false positive measures $)$ ). Positive predictive values are calculated as (true positive measures $) /($ true positive measures $)+($ false positive measures)), and negative predictive value as (true negative measures)/ ((true negative measures )+(falsenegative measures)).

\section{Statistical Analysis}

Data were quantified in $\mathrm{R}$ (The GNU project). For descriptive analyses, we used mean \pm standard deviation, range or median, and interquartile range for continuous values. Differences for the markers and their dependencies on PCC were calculated using multivariate ANOVA analyses. Two-tailed $p$ values less than 0.05 were considered statistically significant. All data were checked for normality using the Shapiro-Wilk test.

\section{RESULTS}

Of the forty healthy subjects included in this study, 24 MPI sessions were excluded because of; non-compliance with the study protocol $(N=4,1$ subject), breath-hold during the low-dose computed tomography scans acquired for attenuation correction purposes (shifting of the myocardium on PET and CT with consequent changes in the anatomical position of diaphragm and lungs, rendering it impossible to obtain acceptable co-registration of the datasets) $(N=5)$, significant motion during the emission scans $(N=4)$, incomplete physiological measures $(N=4)$, and missing emission raw data (listmode data) $(N=7)$. Finally, 7 of the baseline MPI sessions ( $0 \mathrm{mg}$ caffeine ingested) were reported to have $\mathrm{PCC} \geq 1.0 \mathrm{mg} / \mathrm{L}(\mathrm{PCC}=4.3 \pm 3.3 \mathrm{mg}$ / L) and, thus, not considered in this study. In total, 129 of the acquired 160 MPI sessions were deemed acceptable for further studies (80.6\% of the MPI sessions) (0mg MPI: 63, $100 \mathrm{mg}$ : 19, $200 \mathrm{mg}$ : 15, $300 \mathrm{mg}$ : 16, 400 mg: 16). PCC obtained for the five caffeine ingestion protocols is shown in Table 1.

Employing the cohort-specific threshold for normal MFR, adequate hyperemic response was obtained in $95.2 \%$ of the 63 accepted MPI sessions with caffeine concentrations $<1.0 \mathrm{mg} / \mathrm{l}$; and $71.2 \%$ of the 66 accepted MPI sessions with caffeine concentrations $\geq 1.0 \mathrm{mg} / 1$ (Figure 3). In supplementary material 1, we show the normal ranges and the respective cut-off values obtained for the baseline scans (with repeat $\mathrm{PCC}<1.0 \mathrm{mg} / \mathrm{L}$ ) for all seven markers and the normalized MFR values.

MPI sessions with caffeine concentrations $<1.0$ $\mathrm{mg} / \mathrm{l}$

The average PCC was $0.2 \pm 0.2 \mathrm{mg} / \mathrm{l}$ (38 subjects with 63 accepted MPIs) (Table 2). Adequate hyperemic response was observed in $95.2 \%$ (60 out of 63 MPI sessions) when employing the MFR cut-off value (MFR 
Table 1. PCC obtained for MPI sessions with and without consumption of caffeine. $N$ indicates the number of MPI sessions acquired using the respective caffeine doses. Significant differences in the PPC were reported for the two genders for all 5 caffeine consumption groups.

\section{PCC (mg/l)}

\begin{tabular}{|c|c|c|c|c|c|}
\hline & $\begin{array}{c}\text { Omg caffeine } \\
\text { given } \\
(N=63)\end{array}$ & $\begin{array}{l}100 \mathrm{mg} \\
\text { caffeine given } \\
(N=19)\end{array}$ & $\begin{array}{l}200 \mathrm{mg} \\
\text { caffeine given } \\
(N=15)\end{array}$ & $\begin{array}{c}300 \mathrm{mg} \\
\text { caffeine given } \\
(N=16)\end{array}$ & $\begin{array}{c}400 \mathrm{mg} \\
\text { caffeine given } \\
(N=16)\end{array}$ \\
\hline Females & $\begin{array}{l}0.3 \pm 0.2 \\
(N=28)\end{array}$ & $\begin{array}{l}2.7 \pm 0.6 \\
(N=9)\end{array}$ & $\begin{array}{l}6.0 \pm 2.4 \\
(N=7)\end{array}$ & $\begin{array}{l}8.2 \pm 0.1 \\
(N=8)\end{array}$ & $\begin{array}{l}13.1 \pm 5.5 \\
(N=9)\end{array}$ \\
\hline Males & $\begin{array}{l}0.1 \pm 0.2 \\
(N=35)\end{array}$ & $\begin{array}{l}1.4 \pm 0.3 \\
(N=10)\end{array}$ & $\begin{array}{l}5.2 \pm 0.6 \\
(N=8)\end{array}$ & $\begin{array}{l}4.6 \pm 0.9 \\
(N=8)\end{array}$ & $\begin{array}{l}7.0 \pm 1.3 \\
(N=7)\end{array}$ \\
\hline Grouped & $0.2 \pm 0.2$ & $2.0 \pm 0.7$ & $5.5 \pm 2.4$ & $6.4 \pm 2.1$ & $10.4 \pm 5.2$ \\
\hline
\end{tabular}

PCC plasma caffeine concentration

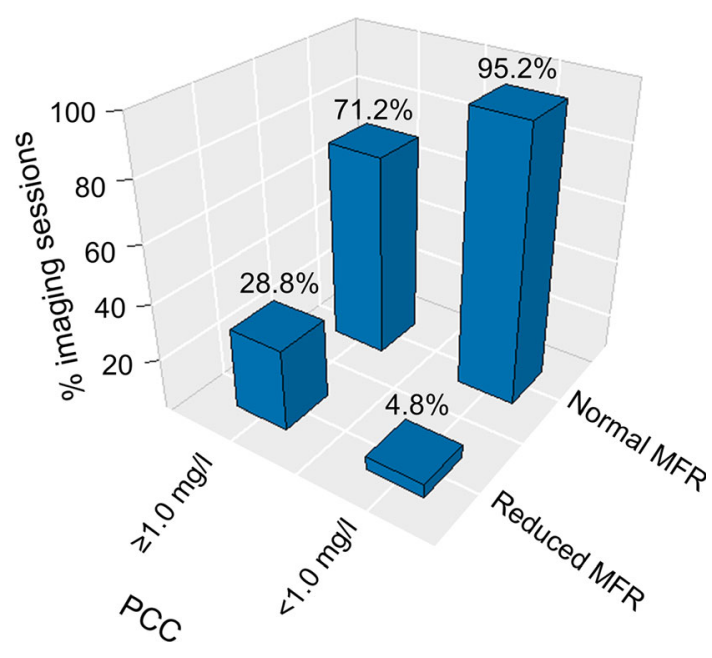

Figure 3. Bar plot of normal vs. reduced MFR according to caffeine concentration. Normal MFR was declared when the MFR was $\geq 68 \%$ of the maximum MFR obtained for the baseline MPIs. MFR myocardial flow reserve, PCC plasma caffeine concentration.

$\geq 68 \%$ of the maximal MFR obtained during the baseline MPI sessions), while adequate hyperemic response was predicted in $81.0 \%$ to $90.5 \%$ of the sessions (range: 51-57 out of 63 MPI sessions) when employing the seven selected markers cut-off values (Table 3). Bar plots of normal versus reduced MFR according to the cut-off value of SSR, SIR, and $\Delta \% \mathrm{HR}$, and stratified to caffeine concentration, are shown in figures 4-6. Similar barplots for $\Delta \mathrm{HR}, \Delta \mathrm{RPP}, \Delta \mathrm{SBP}$, and $\triangle \mathrm{CVR}$ are shown in supplementary material.

MPI sessions with caffeine concentrations $\geq 1.0 \mathrm{mg} /$ 1

The average PCC were $5.9 \pm 4.2 \mathrm{mg} / \mathrm{l}$ (36 subjects with 66 accepted MPIs) (Table 2). Adequate hyperemic response was observed in $71.2 \%$ (47 of 66 MPI sessions) when employing the MFR cut-off value (MFR $\geq 68 \%$ of the maximal MFR obtained during the baseline MPI sessions), while adequate hyperemic response was predicted in $3 \%$ to $84.8 \%$ (range: $2-56$ out of 66 MPI sessions) when employing the seven selected markers cut-off values (Table 3). Bar plots of normal versus reduced MFR according to cut-off value of SSR, SIR, and $\Delta \% \mathrm{HR}$, and stratified to caffeine concentration, are shown in figures 4-6. Similar Bar plots for $\Delta \mathrm{HR}, \Delta \mathrm{RPP}$, and $\Delta \mathrm{CVR}$ are shown in supplementary material 2-4.

\section{Marker Sensitivities and Specificities}

The sensitivity and specificity for all seven markers are shown in Figure $7 \mathrm{a}$, while Figure $7 \mathrm{~b}$ shows the positive and negative predictive values of the markers. Similar sensitivities and specificities were observed for all markers except SRR, which had reduced sensitivity, when the subjects did not have any caffeine intake prior to the MPI, while excellent PPV values were obtained for all markers (Figure 7). However, following caffeine ingestion, the sensitivities dropped for 4 of 7 markers, while SRR had a marginal gain in the sensitivity following caffeine ingestion. The caffeine was observed to reduce the positive predictive value for all seven markers, while the negative predictive values were increased.

\section{DISCUSSION}

This study evaluated the sensitivity and specificity of seven markers suggested to monitor adequate pharmacological stressing of patients when employing adenosine in ${ }^{82} \mathrm{Rb}$ MPI sessions. In this controlled, 
Table 2. MPI measurements and performance of the seven markers used for testing of adequate hyperemic response. Significant differences were observed for four of seven markers when the PCC was increased to $\geq 1.0 \mathrm{mg} / \mathrm{L}$. changing, marked in bold (all $p<0.05$ ).

\begin{tabular}{|c|c|c|}
\hline & $<1.0 \mathrm{mg} / \mathrm{l}$ & $\geq 1.0 \mathrm{mg} / \mathrm{l}$ \\
\hline Number of subjects (MPI sessions) & $38(63)$ & $39(66)$ \\
\hline MFR & $3.80 \pm 0.87$ & $3.39 \pm 1.21(p=0.027)$ \\
\hline PCC (mg/l) & $0.2 \pm 0.2$ & $5.9 \pm 4.2(p<0.001)$ \\
\hline \multicolumn{3}{|l|}{ Stress markers } \\
\hline SRR & $0.59 \pm 0.15$ & $0.70 \pm 0.16(p<0.001)$ \\
\hline SIR & $0.70 \pm 0.13$ & $0.75 \pm 0.14$ \\
\hline$\Delta \mathrm{HR}$ & $29 \pm 13$ & $46 \pm 38(p<0.001)$ \\
\hline$\Delta \% \mathrm{HR}$ & $44 \pm 21$ & $23 \pm 37(p=0.001)$ \\
\hline$\triangle \mathrm{RPP}$ & $3,091 \pm 1,730$ & $1,075 \pm 5080(p=0.003)$ \\
\hline$\triangle \mathrm{SBP}$ & $49 \pm 12$ & $2 \pm 14(p<0.001)$ \\
\hline$\Delta \mathrm{CVR}$ & $69 \pm 26$ & $62 \pm 32$ \\
\hline
\end{tabular}

$P C C$ plasma caffeine concentration, SRR splenic response ratio, SIR splenic stress-to-rest intensity ratio, $\Delta H R$ and $\triangle \% H R$ the change in measured and the percentwise heart rate from rest to stress MPI, respectively. $\triangle R P P$ the change in the rate pressure product from rest to stress MPI. $\triangle S B P$ change in the systolic blood pressure from rest to stress, $\triangle C V R$ the change in the cardiovascular resistance from rest to stress MPI. MFR myocardial flow reserve

Table 3. Number of subjects considered to have adequate pharmacological stressing as a function of the PCC. Numbers in parenthesis indicate percentwise population.

\begin{tabular}{lllllllll}
\hline & SRR & SIR & $\Delta$ HR & $\Delta \%$ HR & $\Delta$ RPP & $\Delta$ SBP & $\Delta$ CVR & MFR \\
\hline$<1 \mathrm{mg} / \mathrm{l}$ & 53 & 51 & 53 & 53 & 54 & 52 & 57 & 60 \\
$(N=63)$ & $(84.1 \%)$ & $(81.0 \%)$ & $(84.1 \%)$ & $(84.1 \%)$ & $(85.7 \%)$ & $(82.5 \%)$ & $(90.5 \%)$ & $(95.2 \%)$ \\
$\geq 1 \mathrm{mg} / \mathrm{l}$ & 40 & 48 & 56 & 38 & 45 & 2 & 54 & 47 \\
$(N=66)$ & $(60.6 \%)$ & $(72.7 \%)$ & $(84.8 \%)$ & $(57.6 \%)$ & $(68.2 \%)$ & $(3.0 \%)$ & $(81.8 \%)$ & $(71.2 \%)$
\end{tabular}

$P C C$ plasma caffeine concentration, $S R R$ splenic response ratio, $S I R$ splenic stress-to-rest intensity ratio, $H R$ heart rate, $R P P$ rate pressure product, $S B P$ systolic blood pressure, $C V R$ cardiovascular resistance, $M F R$ myocardial flow reserve, $\Delta$ changes between rest and stress $\mathrm{MPI}$

randomized study of healthy subjects, sensitivities and specificities were acceptable for all physiological and image-derived markers. The main message from this study is that all the markers for adequate stressing should only be used with care in the clinical routine and only when the patients have followed the guideline recommendations on abstaining from caffeine for a minimum of 12 hours before the stress MPI sessions. ${ }^{14}$

Adequate stressing is of utmost importance to ensure reliable assessments of stress myocardial blood flow and thereby MFR. In this study, the sensitivity and specificity of seven markers were evaluated in a cohort of healthy subjects who underwent four serial MPI sessions with and without caffeine consumption prior to stress MPI (Figure 1). Caffeine (1,3,7 trimethylxanthine) is thought to competitively inhibit the adenosine receptor and thus may also attenuate the hyperemic cardiac response following a dose-dependent affinity with gender-specific responses. ${ }^{7,9,15}$ Reduced cardiac hyperemia is expected for patients when plasma caffeine concentrations $\geq 1.0 \mathrm{mg} / \mathrm{l}$ when adenosine, regadenosone or dipyridamole are used as pharmaceutical stressing agents owing to caffeine's non-specific binding to the $\mathrm{A}_{2 \mathrm{~A}}$ receptor. ${ }^{4,7-9,14,16-18}$ In this context, the PCC level affects the hyperemic response more strongly in men than females. ${ }^{7}$ In this study, reduced hyperemic responses were observed in $22(16.2 \%)$ of the 129 accepted MPI sessions, of which 19 (86.4\%) had elevated PPC (Table 3). For SRR, adequate stressing was called when the ratio was $\leq 0.75$, obtained as the activity observed in the spleen normalized to the activity observed in the liver. This phenomenon occurs as adenosine receptor $A_{1}$ stimulation reduces splanchnic artery perfusion resulting in splenic blood flow 

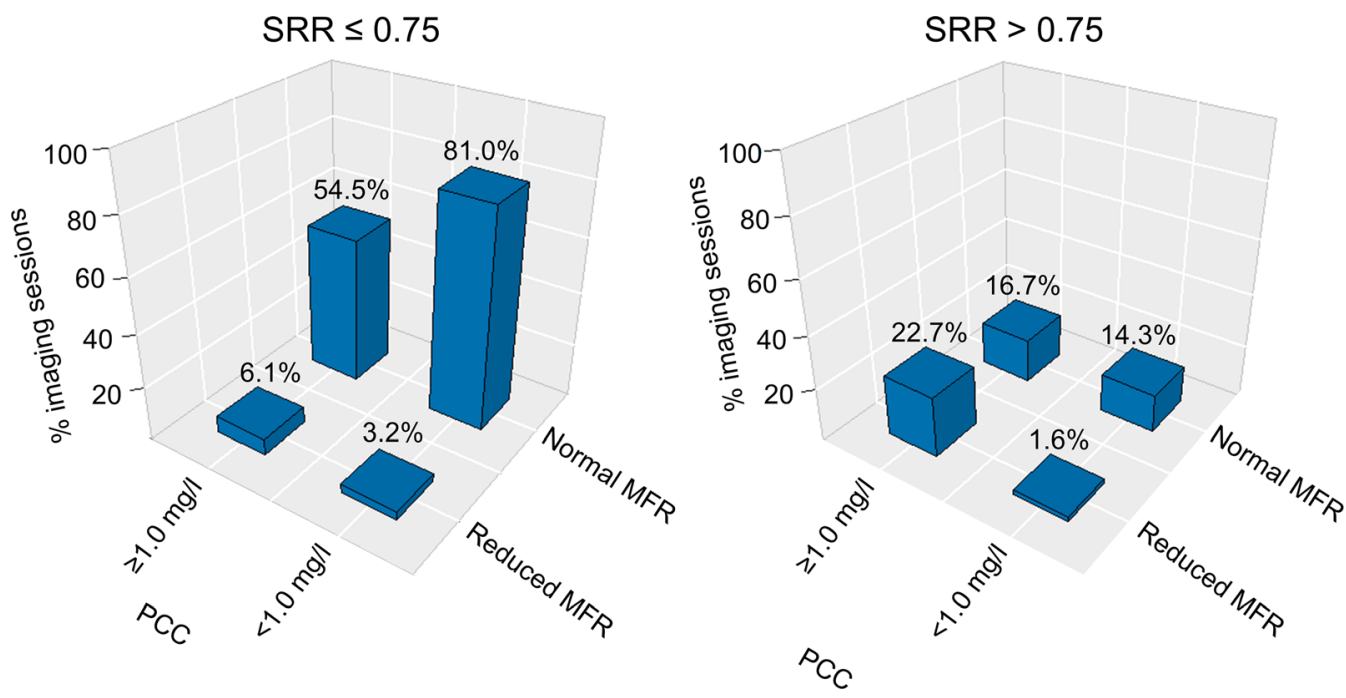

Figure 4. Bar plot of studies with and without adequate hemodynamic response when employing SSR, stratified by PPC. Adequate hemodynamic response was declared for SRR $\leq 0.75$. The numbers above bars indicate the percentwise number of occurrences for the respective measures. $S R R$ splenic response ratio, $M F R$ myocardial flow reserve, $P C C$ plasma caffeine concentration.
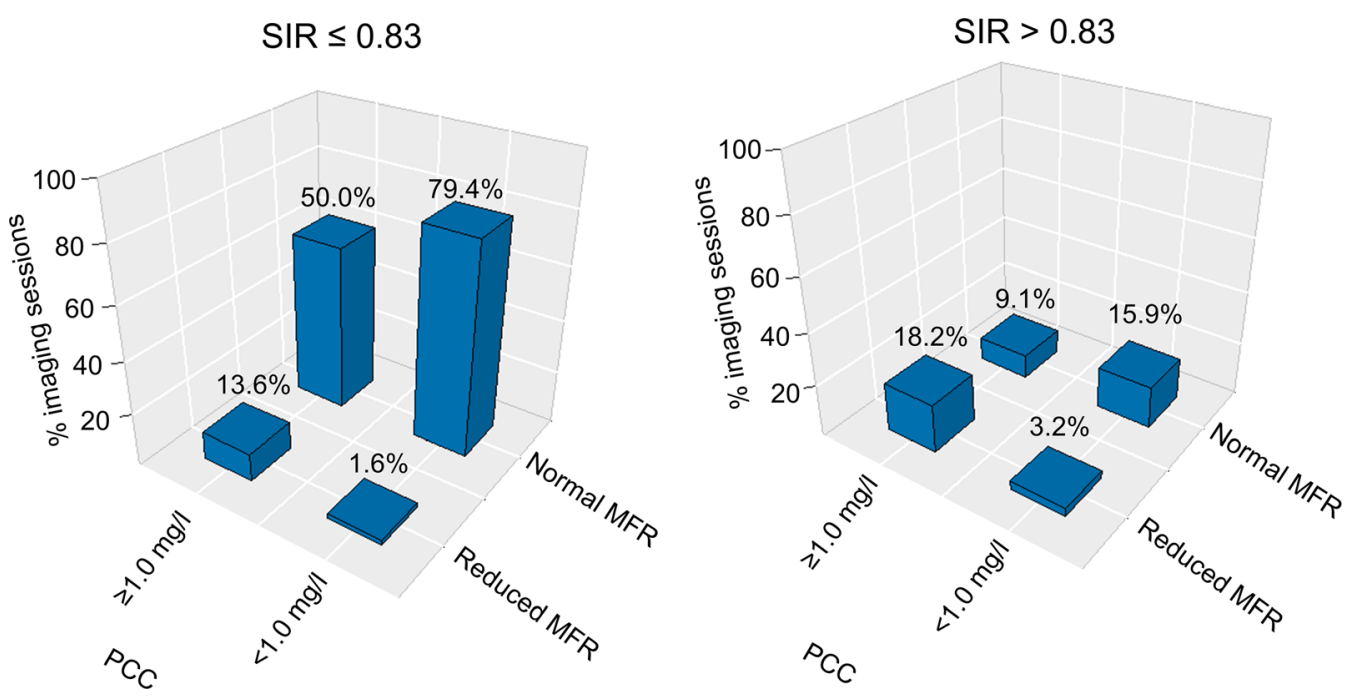

Figure 5. Bar plot of studies with and without consideration of adequate hemodynamic response when employing SIR. Adequate hemodynamic response was declared for $\operatorname{SIR} \leq 0.83$. The numbers above bars indicate the percentwise number of occurrences for the respective measures. The data were divided into two subgroups, studies with and without significant plasma concentrations of caffeine (PCC $\geq 1.0 \mathrm{mg} / \mathrm{l}$ and $\mathrm{PCC}<1.0 \mathrm{mg} / \mathrm{l}$ ), respectively. SIR splenic stress-to-rest intensity ratio, $M F R$ myocardial flow reserve, $P C C$ plasma caffeine concentration, $M P I$ myocardial perfusion imaging.

attenuation. For this approach to be successful, it demands that adenosine and caffeine have identical pharmacological potency at the $\mathrm{A}_{1}$ and $\mathrm{A}_{2 \mathrm{~A}}$ receptor sites. This was not the case in our study, with a modest correlation between cardiac hyperemia $\left(\mathrm{A}_{2 \mathrm{~A}}\right)$ and reduced splenic perfusion $\left(A_{1}\right)$. Similarly, the same reservation applies to the SIR estimate.

The ability of adenosine to induce tachycardia is ascribed to peripheral vasodilatation, with the assumption that HR change is a direct reflection of peripheral 

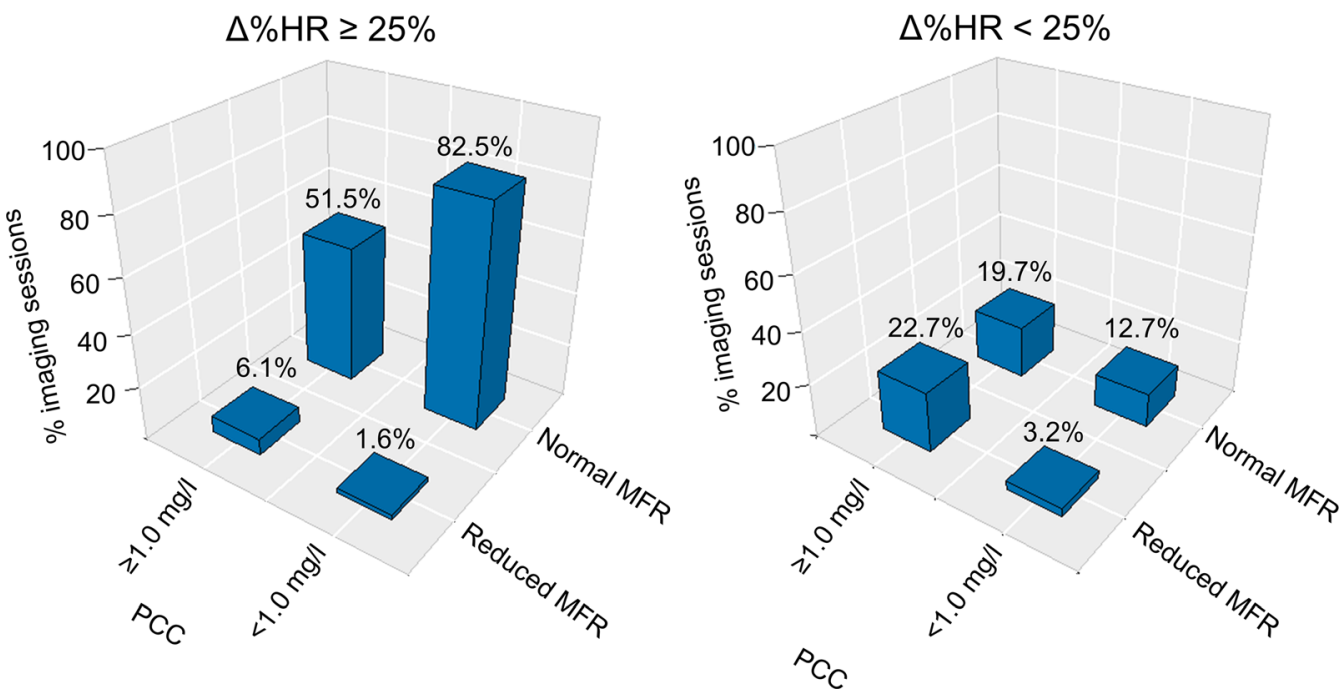

Figure 6. Bar plot of studies with and without consideration of having adequate hemodynamic response for $\Delta \% \mathrm{HR}$, stratified by PPC. Adequate hemodynamic response was declared for $\Delta \% \mathrm{HR} \geq 25$. The numbers above bars indicate the percentwise number of occurrences for the respective measures. $\triangle \% H R$ percent wise change in peak heart rate as observed for the ECG trigger signals between rest and stress MPI, MFR myocardial flow reserve, $P C C$ plasma caffeine concentration, MPI myocardial perfusion imaging.

vasodilatation. In addition, both direct and reflex baroreceptor-mediated sympathetic activation is thought to play a role. ${ }^{19,20}$ In a previous study it was found that a change in $\mathrm{HR}>15$ beats per minute was associated with adequate stressing of the patients - a threshold lower than reported in this study $(\Delta \mathrm{HR} \geq 18) .^{21}$ The discrepancy in the $\Delta \mathrm{HR}$ reported in this study when compared to Kotecha et al's results $\left({ }^{21}\right)$ might be associated with the differences in the age of the study cohorts evaluated and reflects that $\Delta \mathrm{HR}$ alone might not be fully representative for adequate stressing of the patients. However, the percentwise change in the HR provided the better sensitivity and specificity, while they had predictive values compared to $\Delta \mathrm{HR}$. In this study, an increase of $\geq 25 \%$ of the rest HR was a positive predictor that the subjects had adequate stressing during the scans. Likewise, changes in the RPP were evaluated as a marker for adequate stressing of the subjects. An increase in the RPP $\geq 1250$ was found to be an acceptable predictor of adequate stressing during the MPI sessions. Adenosine results in a modest increase in heart rate and a modest decrease in both systolic and diastolic BPs. ${ }^{22}$ Reductions in the SBP from rest to stress were evaluated as a marker for adequate stressing of the patients. It was found that it had an excellent sensitivity to elevated PCC values; however, it had a poor specificity to detect sufficient hyperemic response in the volunteers when $\Delta \mathrm{SBP}>38$ was used as a cut-off value. Finally, $\triangle \mathrm{CVR}$ was found to offer the poorest sensitivity and specificity when caffeine consumption could not be ruled out. In this study, adequate stressing of the patients was declared when $\triangle \mathrm{CVR} \geq 40 \%$ in the rest scan as compared to the stress scan.

In this study, both SRR and SIR measures were in concordance with previous findings. ${ }^{21,23}$ Although all evaluated markers serve as acceptable indicators for adequate stress, the cut-off values are found to vary across studies. $^{12,21,23}$ The varying cut-off values indicate that the thresholds employed are cohort-specific and, thus, a generalization of the methods is difficult. Moreover, the non-consistent cut-off values are a general limitation of all the suggested techniques, which also applies to this study. Another limitation to this study is the small cohort evaluated, consisting of 40 volunteers. Linked to the small cohort is that the volunteers are young, healthy individuals and thus not the typical patient undergoing MPI, with an intermediate likelihood of ischemic heart, which might affect the sensitivity and specificity of the markers. Finally, any cox-hazard models could not be calculated for the respective methods in this study, and, thus, the diagnostic impact of the methods cannot be determined. 

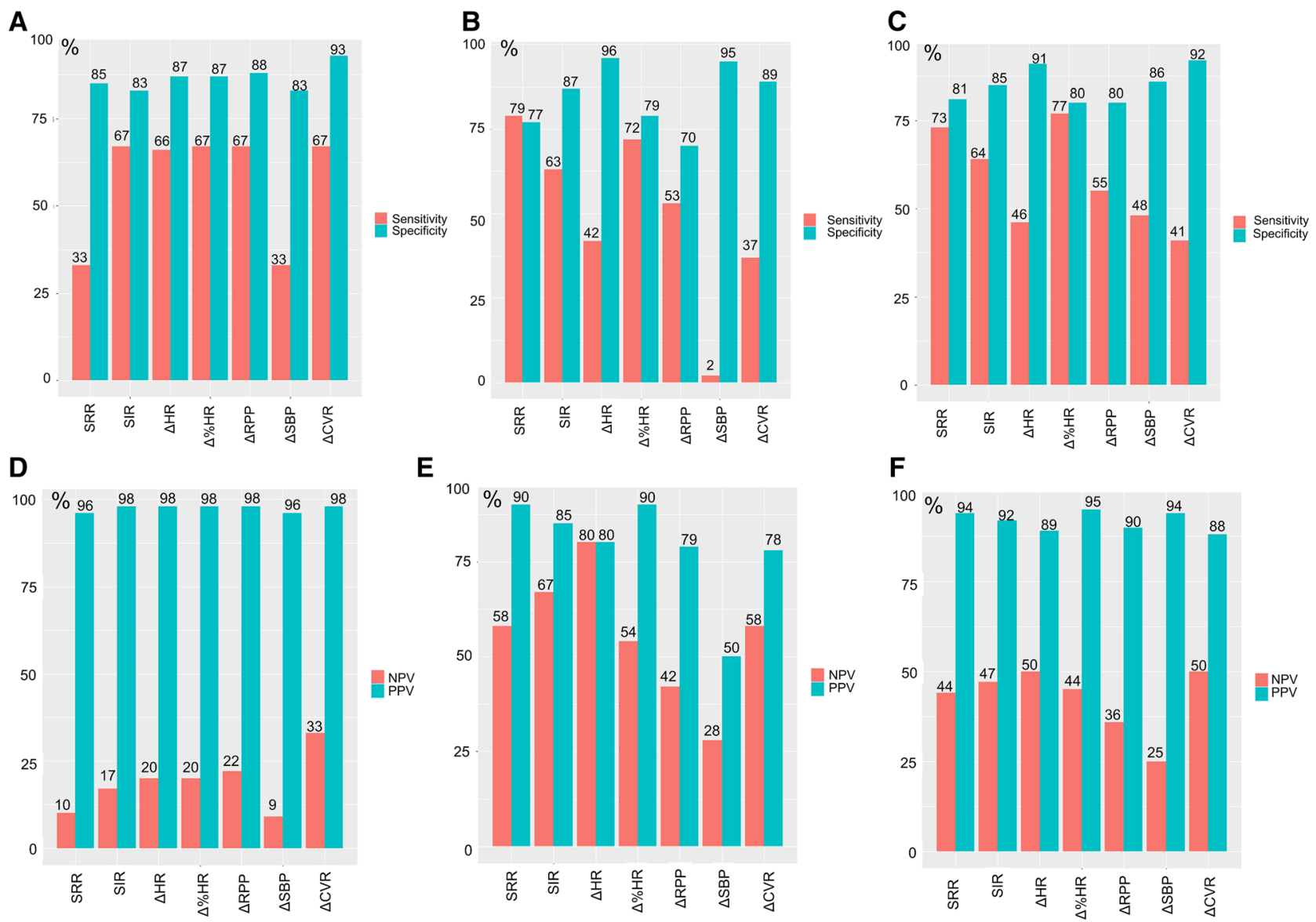

Figure 7. Performance measures for the seven markers used for identification of adequate stressing of the volunteers. Sensitivity and specificity for the data obtained with $\mathrm{PCC}<1.0$ and $\mathrm{PCC} \geq 1.0 \mathrm{mg} / \mathrm{l}$ are shown in $\mathbf{a}$ and $\mathbf{b}$ respectively. The grouped measures (PCC $<1.0$ and $\mathrm{PCC} \geq 1.0 \mathrm{mg} / \mathrm{l})$ are shown in $\mathbf{c}$ PPV and NPV are shown for the datasets with $\mathrm{PCC}<1.0 \mathrm{mg} / \mathrm{L}$ and $\mathrm{PCC} \geq 1.0 \mathrm{mg} / \mathrm{l}$ in $\mathbf{d}$ and $\mathbf{e}$ respectively. In $\mathbf{f}$ the NPV and PPV are shown for the grouped measures $(\mathrm{PCC}<1.0$ and $P C C \geq 1.0 \mathrm{mg} / \mathrm{l})$. $S R R$ splenic response ratio, $S I R$ splenic stress-to-rest intensity ratio, $\Delta H R$ change in heart rate between rest and stress MPI, $\triangle \% H R$ percentwise change in heart rate between rest and stress MPI, $\triangle R P P$ change in rate pressure product, $\triangle S B P$ change in systolic blood pressure, $\triangle C V R$ change in cardiovascular resistance between rest and stress MPI. NPP negative predictive value, $P P V$ positive predictive value.

\section{NEW KNOWLEDGE GAINED}

In this study, seven markers suggested for the detection of adequate hyperemic cardiac response was tested in a controlled setting, with cross-validation of the respective models. Common for all the markers is that they only provide acceptable sensitivities and specificities in a cohort of young volunteers. Therefore, their use warrants care in the clinical setting.

\section{CONCLUSION}

In a cohort of young, healthy individuals, all imagederived and physiological markers provided acceptable sensitivities and specificities when the subjects followed the guideline-recommended caffeine pausation before MPI. Nevertheless, their use warrants great care when caffeine consumption prior to MPI cannot be ruled out.

\section{Acknowledgement}

This project received funding from the European Union's Horizon 2020 research and innovation programme under grant Agreements No. 670261 (ERC Advanced Grant) and 668532 (Click-It), the Lundbeck Foundation, the Novo Nordisk Foundation, the Innovation Fund Denmark, the Danish Cancer Society, Arvid Nilsson Foundation, the Neye Foundation, the Research Foundation of Rigshospitalet, the Danish National Research Foundation (Grant 126), the Research Council of the 
Capital Region of Denmark, the Danish Health Authority, and the John and Birthe Meyer Foundation and Research Council for Independent Research. Andreas Kjaer is a Lundbeck Foundation Professor. No other conflicts of interests exist to this submission.

\section{Open Access}

This article is licensed under a Creative Commons Attribution 4.0 International License, which permits use, sharing, adaptation, distribution and reproduction in any medium or format, as long as you give appropriate credit to the original author(s) and the source, provide a link to the Creative Commons licence, and indicate if changes were made. The images or other third party material in this article are included in the article's Creative Commons licence, unless indicated otherwise in a credit line to the material. If material is not included in the article's Creative Commons licence and your intended use is not permitted by statutory regulation or exceeds the permitted use, you will need to obtain permission directly from the copyright holder. To view a copy of this licence, visit http://creativecommons.org/licenses/by/4.0/.

\section{References}

1. Bateman TM, Dilsizian V, Beanlands RS, et al. American society of nuclear cardiology and society of nuclear medicine and molecular imaging joint position statement on the clinical indications for myocardial perfusion PET. J Nucl Med 2016;57:16546. https://doi.org/10.2967/jnumed.116.180448.

2. Dilsizian V, Bacharach SL, Beanlands RS, et al. ASNC imaging guidelines/SNMMI procedure standard for positron emission tomography (PET) nuclear cardiology procedures. J Nucl Cardiol 2016;23:1187-226. https://doi.org/10.1007/s12350-016-0522-3.

3. Rischpler C, Totzeck M. Are you stressed? J Nucl Cardiol 2019;26:1898-900. https://doi.org/10.1007/s12350-018-1332-6.

4. Sampson UK, Dorbala S, Limaye A, et al. Diagnostic accuracy of Rubidium-82 myocardial perfusion imaging with hybrid positron emission tomography/computed tomography in the detection of coronary artery disease. J Am Coll Cardiol 2007;49:1052-8. h ttps://doi.org/10.1016/j.jacc.2006.12.015.

5. Kidambi A, Sourbron S, Maredia N, et al. Factors associated with false-negative cardiovascular magnetic resonance perfusion studies: a clinical evaluation of magnetic resonance imaging in coronary artery disease (CE-MARC) substudy. J Magn Reson Imaging 2016;43:566-73. https://doi.org/10.1002/jmri.25032.

6. Al Jaroudi W, Iskandrian AE. Regadenoson: a new myocardial stress agent. J Am Coll Cardiol 2009;54:1123-30.

7. Lassen ML, Byrne C, Sheykhzade M, et al. Gender differences and caffeine impact in adenosine-induced hyperemia. J Nucl Med 2021. https://doi.org/10.2967/jnumed.121.261970.

8. Mishra RK, Dorbala S, Logsetty G, et al. Quantitative relation between hemodynamic changes during intravenous adenosine infusion and the magnitude of coronary hyperemia: implications for myocardial perfusion imaging. J Am Coll Cardiol 2005;45:553-8.

9. Byrne C, Kjaer A, Wissenberg M, et al. Dose-dependent effect of caffeine on adenosine-induced myocardial stress perfusion in
Rubidium-82 positron-emission tomography/computed tomography. JACC Cardiovasc Imaging 2019;12:1102-3.

10. Lortie M, Beanlands RSB, Yoshinaga K, et al. Quantification of myocardial blood flow with $82 \mathrm{Rb}$ dynamic PET imaging. Eur J Nucl Med Mol Imaging 2007;34:1765-74. https://doi.org/10.1007/ s00259-007-0478-2.

11. Manisty C, Ripley DP, Herrey AS, et al. Splenic switch-off: a tool to assess stress adequacy in adenosine perfusion cardiac MR imaging. Radiology 2015;276:732-40.

12. Bami K, Tewari S, Guirguis F, et al. Prognostic utility of splenic response ratio in dipyridamole PET myocardial perfusion imaging. J Nucl Cardiol 2019;26:1888-97. https://doi.org/10.1007/s12350018-1269-9.

13. Murthy VL, Bateman TM, Beanlands RS, et al. Clinical quantification of myocardial blood flow using PET: joint position paper of the SNMMI cardiovascular council and the ASNC. J Nucl Med 2018;59:273-93. https://doi.org/10.2967/jnumed.117.201368.

14. Sciagrà R, Lubberink $M$, Hyafil $F$, et al. EANM procedural guidelines for PET/CT quantitative myocardial perfusion imaging. Eur J Nucl Med Mol Imaging 2020. https://doi.org/10.1007/s002 59-020-05046-9.

15. Fredholm BB, IJzerman AP, Jacobson KA, et al. International union of pharmacology. XXV. Nomenclature and classification of adenosine receptors. Pharmacol Rev 2001;53:527-52.

16. Klotz KN. Adenosine receptors and their ligands. Naunyn Schmiedebergs Arch Pharmacol 2000;362:382-91. https://doi.org/10. 1007/s002100000315.

17. Kitkungvan D, Bui L, Johnson NP, et al. Quantitative myocardial perfusion positron emission tomography and caffeine revisited with new insights on major adverse cardiovascular events and coronary flow capacity. Eur Heart $\mathrm{J}$ Cardiovasc Imaging 2019;20:751-62. https://doi.org/10.1093/ehjci/jez080.

18. White JR, Padowski JM, Zhong Y, et al. Pharmacokinetic analysis and comparison of caffeine administered rapidly or slowly in coffee chilled or hot versus chilled energy drink in healthy young adults. Clin Toxicol 2016;54:308-12. https://doi.org/10.3109/155 63650.2016 .1146740

19. Alberti C, Monopoli A, Casati C, et al. Mechanism and pressor relevance of the short-term cardiovascular and renin excitatory actions of the selective A2A-adenosine receptor agonists. J Cardiovasc Pharmacol 1997;30:320-4.

20. Biaggioni I, Killian TJ, Mosqueda-Garcia R, et al. Adenosine increases sympathetic nerve traffic in humans. Circulation 1991;83:1668-75.

21. Kotecha T, Monteagudo JM, Martinez-Naharro A, et al. Quantitative cardiovascular magnetic resonance myocardial perfusion mapping to assess hyperaemic response to adenosine stress. Eur Hear Journal-Cardiovascular Imaging 2020;22:273-81.

22. Cerqueira MD, Verani MS, Schwaiger M, et al. Safety profile of adenosine stress perfusion imaging: results from the adenoscan multicenter trial registry. J Am Coll Cardiol 1994;23:384-9.

23. Patriki D, von Felten E, Bakula A, et al. Splenic switch-off as a predictor for coronary adenosine response: validation against $13 \mathrm{~N}$ ammonia during co-injection myocardial perfusion imaging on a hybrid PET/CMR scanner. J Cardiovasc Magn Reson 2021;23:1-7. https://doi.org/10.1186/s12968-020-00696-y.

Publisher's Note Springer Nature remains neutral with regard to jurisdictional claims in published maps and institutional affiliations. 